\title{
Filamentary nanosecond surface dielectric barrier discharge. Experimental comparison of the streamer-to-filament transition for positive and negative polarities.
}

\author{
Ch. Ding, A. Yu. Khomenko, S. A. Shcherbanev, \\ S. M. Starikovskaia \\ Laboratory of Plasma Physics (CNRS, Ecole Polytechnique, Univ. Paris-Sud, \\ Observatoire de Paris, Sorbonne Universitée, l'Institut Polytechnque de Paris), Ecole \\ Polytechnique, route de Saclay, 91128 Palaiseau, France \\ E-mail: svetlana.starikovskaia@lpp.polytechnique.fr
}

\begin{abstract}
Streamer-to-filament transition is a general feature of high pressure high voltage nanosecond surface dielectric barrier discharges (nSDBDs) for mixtures containing molecular gases. The transition is observed at high pressures and voltages in a single-shot experiment a few nanoseconds after the start of the discharge. A set of experimental results comparing streamer-to-filament transition and properties of plasma in the filaments for the identical high voltage pulses of negative and positive polarity is presented. The transition curves in voltage-pressure coordinates are obtained for $\mathrm{N}_{2}: \mathrm{O}_{2}$ mixtures with different content of molecular oxygen, from 0 to $20 \%$, at the pressure range 1-12 bar. Continuous optical spectra are compared for both polarities in 6 bar synthetic air. Electron density is calculated from Stark broadening of $\mathrm{H}_{\alpha}$ line at $\lambda=656.5 \mathrm{~nm}$ in the discharge and in early afterglow, 40 nanoseconds after the end of the high voltage pulse. Hydrodynamic perturbations are measured using schlieren imaging in 1-6 bar air for streamer and filamentary mode for both polarities. The review of common and distinctive features of the filamentary single-shot nSDBD for two polarities of the applied pulse is provided.
\end{abstract}

Keywords: streamer-to-filament transition, nanosecond surface dielectric barrier discharge 


\section{Introduction}

Nanosecond surface dielectric barrier discharges (nSDBDs) at atmospheric pressure have been studied extensively over the last two decades for flow control [1-5]. Plasma of nanosecond DBDs is close by parameters to plasma of volumetric pulsed streamer discharge, with a dominant excitation of electronic levels of molecules [6]. Synchronous start, within $0.2 \mathrm{~ns}$, of the streamers from the edge of the high-voltage electrode and propagation along the dielectric provide a synchronous energy relaxation with generation of weak shock waves near the surface of the dielectric. Numerical modeling of the nSDBDs developed during last 10 years, provides a deep insight into physics of the nanosecond surface discharges [7-9].

The nSDBD was suggested as a source for plasma-assisted ignition of combustible mixtures in $[10,11]$. During the experiments at high pressures [12-14], it was found that a severe transformation of a single-shot nanosecond surface streamer discharge is observed at increasing gas pressure and/or voltage. A regular structure of tens of filaments appeared from the edge of the high-voltage electrode and propagated in the direction perpendicular to the edge of the high-voltage electrode, the number of filaments being $4-5$ times less than the number of streamers. The effect was first observed for negative polarity discharge [12] and later it was found that the streamer-to-filament transition is a common feature for all observed molecular gases and mixtures containing molecular gases [13]. Extremely high electron density, $n_{e}=10^{18}-10^{19} \mathrm{~cm}^{-3}$ and slow electron density decay, tens of nanoseconds, were reported for $\mathrm{N}_{2}: \mathrm{H}_{2}$ mixture [14]. During last $3-5$ years, a few groups [15-17] observed similar high densities of electrons with a slow decay rate in atmospheric pressure plasma driven by repetitive nanosecond pulses; constriction of the discharge corresponding to sharp increase of the electron density in a nanosecond time scale has been experimentally observed.

In the case of the volumetric discharge, filaments can appear near both electrodes closing in the center of the discharge gap; the distance between the electrodes is typically not longer than hundreds of microns. In the case of the surface discharge, the filaments start always from a single open high-voltage electrode, and so negative and positive polarity filaments can be easily distinguished experimentally. To provide a material for further analysis and theoretical research, the present paper summarizes and systemizes our observations comparing filamentary discharges for different polarities.

\section{Experimental setup}

The coaxial electrode system is presented in figure 1 (a) and described elsewhere $[13,14]$. A metal disc $20 \mathrm{~mm}$ in diameter served as a $\mathrm{HV}$ electrode during the majority of the experiments. For the set of experiments on optical emission spectra, the disk has been purposely replaced by a same diameter gear, what would be discussed in the "Results and discussion" section. The internal diameter of the low-voltage grounded electrode was equal to the diameter of the high voltage electrode, and the external diameter of 
Filamentary nanosecond SDBD. Comparison of both polarities.
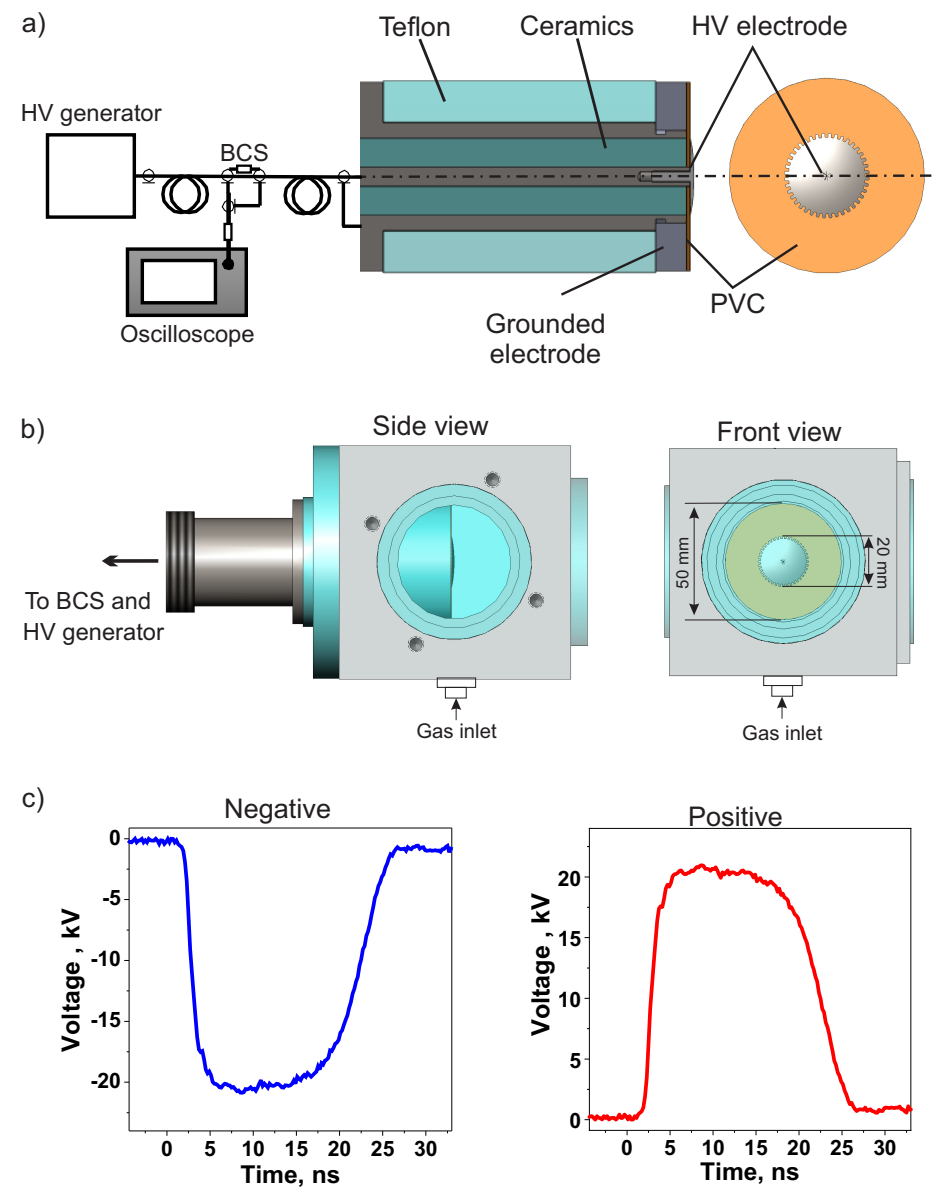

Figure 1. Schematic of the experimental apparatus: (a) cylindrical electrode system; (b) high-pressure chamber with mounted electrode system; (c) typical voltage on the electrode for pulses of negative and positive polarity

the low voltage electrode was equal to $50 \mathrm{~mm}$. A thin $0.3 \mathrm{~mm}$ dielectric layer (PVC, $\varepsilon \approx 3-3.5)$ was glued to the grounded electrode by Geocel FIXER Mate silicon glue $(\varepsilon \approx 3)$, the thickness of the glue layer was $0.4 \pm 0.1 \mathrm{~mm}$, giving the total thickness of the dielectric equal to $0.7 \pm 0.1 \mathrm{~mm}$.

The electrode system was mounted into a constant volume high pressure chamber with 3 quartz optical windows $5 \mathrm{~cm}$ in diameter (see figure 1 (b)). One optical window was situated in front of the electrode. The images of the discharge as well as the optical emission spectra were acquired through the front window. Two other windows allowed the observation of the discharge from the side and were used for schlieren diagnostics.

The discharge chamber was pumped down to $\sim 10^{-3}$ Torr before the experiments; nitrogen and oxygen (Air Liquide) with $<100$ ppm of impurities were used to prepare the mixtures. The experiments were performed in a single shot regime at ambient initial temperature in pure $\mathrm{N}_{2}$ and in mixtures containing 1, 2, 5, 10 and $20 \%$ of $\mathrm{O}_{2}$ in nitrogen by partial pressure.

Identical in shape positive or negative polarity high voltage pulses, 20 ns in duration and $2 \mathrm{~ns}$ rise time, $15-60 \mathrm{kV}$ in amplitude on the high voltage electrode were delivered 
by a $30 \mathrm{~m}$ long coaxial $50 \Omega$ cable connecting the high pressure chamber and the FPG2003PM or FPG20-03PN pulsers (FID Technology). Typical waveforms for the pulses on the electrode are given in figure 1 (c).

The voltage waveforms were obtained with the help of calibrated back current shunt installed in the middle of the cable, the technique is described in details elsewhere [18]. Current through plasma was calculated subtracting the capacitive current from the total current measured by the back current shunts. The capacitive current was measured experimentally at the conditions when the discharge is absent similar to $[12,13]$. The images $(\lambda=250-800 \mathrm{~nm})$ were taken by a Pi-Max4 Princeton Instruments ICCD camera. The images were used to study the moment of the streamer-to-filament transition as well as to follow the transformation of the discharge with respect to pressure and/or voltage. Broad band optical spectra in the range $\lambda=250-500 \mathrm{~nm}$ and the optical emission at $630-680 \mathrm{~nm}$ were recorded by an ACTON spectrometer (SP-7500i, $600 \mathrm{I} / \mathrm{mm}$ and $1200 \mathrm{I} / \mathrm{mm}$ gratings) and the ICCD camera. A classical two-lens schlieren imaging system synchronized with the discharge [19] was used for visualising the hydrodynamic perturbations produced by the discharge. The Thorlabs LED M505L3-C with a $505 \mathrm{~nm}$ central wavelength and $30 \mathrm{~nm}$ bandwidth powered by microsecond pulses was used as a light source.

\section{Results and discussion}

Streamer-to-filament transition happens in a similar way in all observed experimental conditions. In air at 1 bar a "regular" streamer nSDBD is observed. With the pressure increase, the discharge propagation length during the pulse decreases significantly: for example, in air at 5 bar the propagation length is 5 to 6 times smaller than at 1 bar. When voltage and/or pressure is increased, the discharge appearance changes: a regular structure of discharge channels, 4-5 times rarer than for streamers and much brighter (the light emission increases by a factor of 50 when passing to the filamentary mode) starts near the high voltage electrode, whatever the applied voltage polarity is, and propagates in a radial direction. The filament propagation length is a function of the amplitude and polarity of the applied voltage, the gas pressure and the gas mixture composition.

\subsection{Different behavior of discharge at different polarity: transition voltage}

To build a phase diagram of the streamer-to-filament transition in a Pressure-Voltage chart, the camera gate was fixed to 12 ns. Time-integrated single-shot images of the discharge were taken at constant pressure increasing and decreasing the voltage. It should be pointed out here that no hysteresis was observed by changing the voltage up and down or vise versa. Typical ICCD images of the streamer-to-filament transition are presented in figure ??.

The images were taken for nSDBDs of positive polarity in nitrogen at 6 bar and 
in in air at 7 bar. The relatively low voltage of $\pm 15-20 \mathrm{kV}$ at the high-voltage electrode initiates a streamer nSDBD. Then the voltage increases gradually for each new experiment. When only a few (typically 3-5) filaments are observed during first 12 ns of the discharge, this voltage is considered as a transition one (like the center images in figure 2). It should be noted that (i) from experiment to experiment, the filaments appear at different points on the high-voltage electrode, they are not associated with the defects on the electrode; (ii) the duration of the transition in the developed filamentary mode, that is the time between the first filament appears and when a regular filamentary pattern is observed (similar to the images at $+43 \mathrm{kV}$ and $-47 \mathrm{kV}$ in figure 2), is equal to $1-2$ ns.
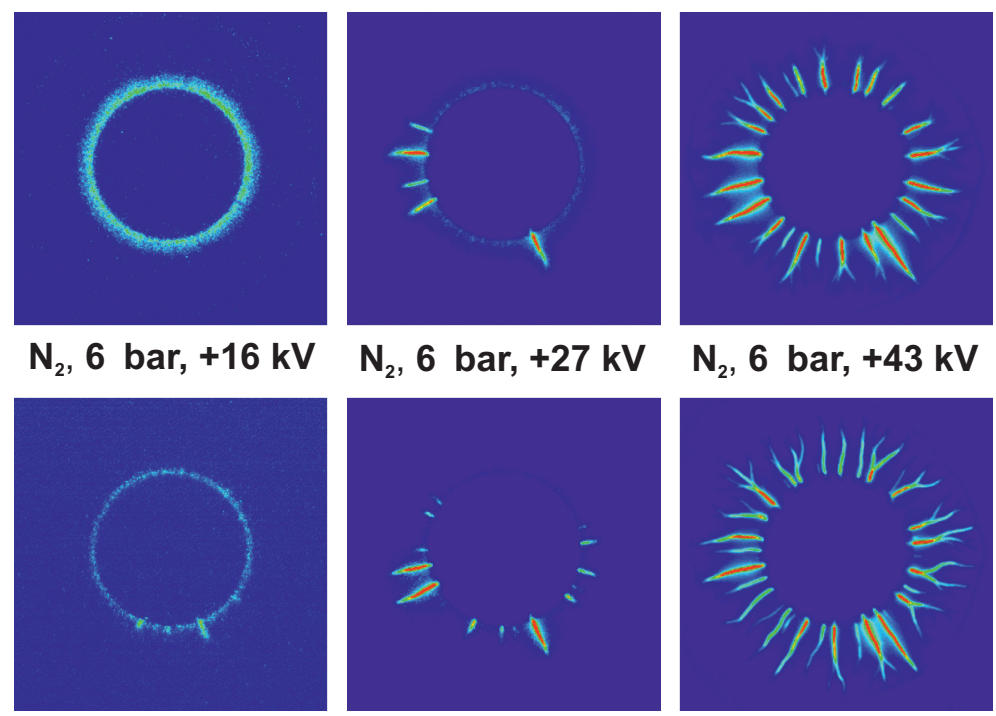

Air, 7 bar, $-19 \mathrm{kV}$

Air, 7 bar, -26 kV Air, 7 bar, -46 kV

Figure 2. Images of streamer surface discharge, transitive discharge and developed filamentary discharge. Upper row: Nitrogen, $P=6$ bar, positive polarity; lower row: air, $P=7$ bar, negative polarity. Camera gate is $12 \mathrm{~ns}$. The diameter of the highvoltage electrode (a circle at the center of each image) is equal to $20 \mathrm{~mm}$.

The most significant difference observed for the filamentary discharge at negative and positive polarities is a behavior of the transition curve. The pressure-voltage charts for both polarities obtained as described above are given by figure 3 . Two set of measurements are compared at each plot: the solid lines represent the results of the present work, where the overall thickness of the PVC layer and the glue layer is 0.7 $\mathrm{mm}$, and the dashed lines show our previous measurements [13], taken at $0.5 \mathrm{~mm}$ of the overall dielectric thickness. The voltage measurement error is less than $1 \mathrm{kV}$.

For negative polarity the streamer-to-filament transition happens almost simultaneously for all observed gases: the difference between air and pure nitrogen is about $5 \mathrm{kV}$ in comparison to tens of kilovolts for positive polarity. There is a point of intersection of curves corresponding to different gas composition, this is especially clearly seen for the data set with four different percentages of oxygen in the mixture. The intersection point seems to be a function of the overall dielectric layer thickness. 
For positive polarity, the effect of molecular oxygen addition is extremely strong: $1 \%$ of oxygen at 4 bar increases the transition voltage from $41 \mathrm{kV}$ to $52 \mathrm{kV}$. This dependence seems to disappear with oxygen concentration rising: at 6 bar, the transition voltages for pure nitrogen and mixtures with 1, 2, 5 and $20 \%$ of oxygen are equal to $30,40,44$, 48.5 and $49.5 \mathrm{kV}$ respectively.
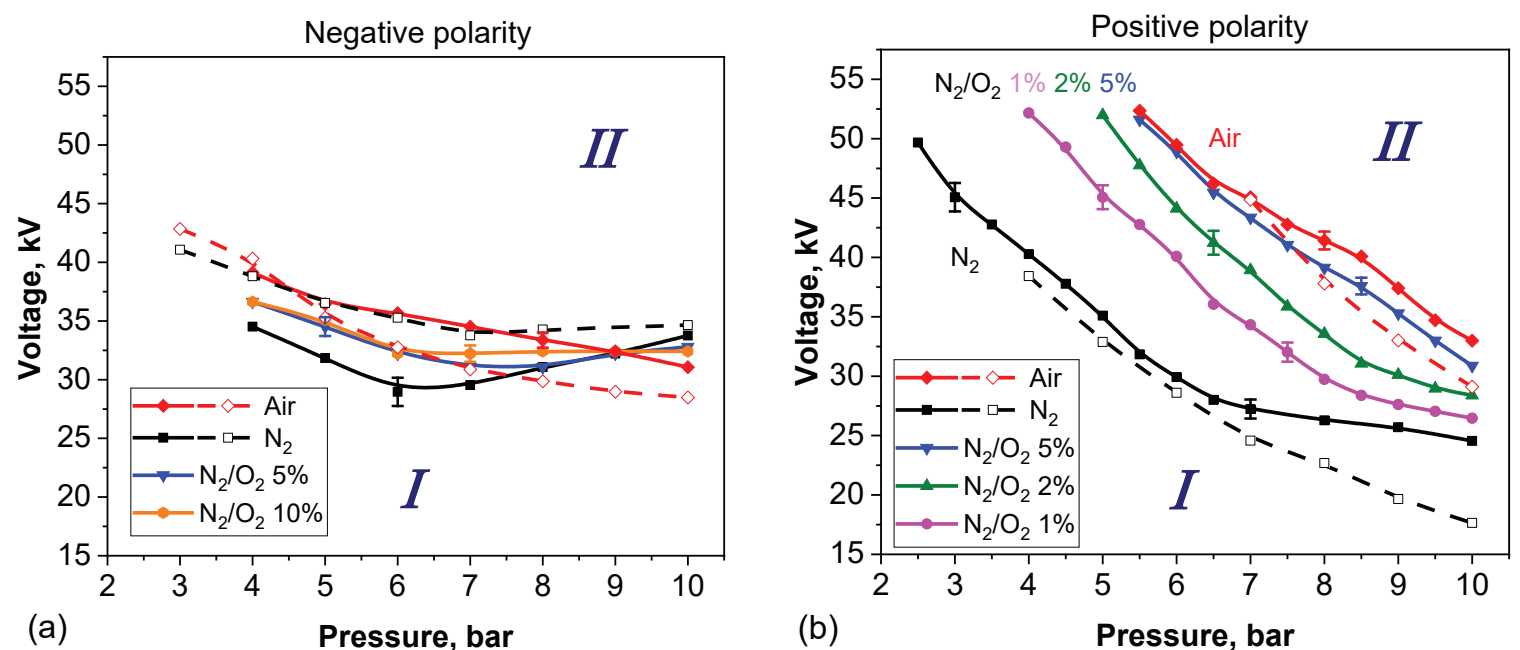

Figure 3. Curves of streamer-to-filament transition taken at the condition that the transition happens during first 12 ns of the discharge for (a) negative and (b) positive polarity of voltage on the high-voltage electrode.

The time instant of the filamentation (figure 4) is in a good agreement with the observed $(\mathrm{P} ; \mathrm{V})$-diagram. The transition time was measured with the ICCD gate of $0.5 \mathrm{~ns}$ as the time instant of 2-3 filaments appearing near the high-voltage electrode. It is clearly seen that the time of the filamentation decreases with pressure. It is also seen that for the negative polarity, the time of filamentation is almost the same for $\mathrm{N}_{2}$ and air, while for the positive polarity, the time of filamentation in air is $2-4$ times longer than in $\mathrm{N}_{2}$. One of the hypotheses is that in the mixtures containing $\mathrm{O}_{2}$, the efficient photoionization provides a stability of streamer plasma for longer period.

Below any of the transition curves (region $I$ in figure 3) for any observed mixtures the streamer plasma is similar to the SDBD plasma at atmospheric pressure: its optical spectrum contains mainly the nitrogen second positive system, with less pronounced emission of the first negative (in the front) and the first positive (behind the front) systems; the electron density is around $n_{e} \approx 10^{14}-10^{15} \mathrm{~cm}^{-3}[8,9]$, the propagation velocity is a few millimeters per nanosecond on the front of the pulse decreasing to $0.1 \mathrm{~mm} / \mathrm{ns}$ for negative and $0.4-0.5 \mathrm{~mm} / \mathrm{ns}$ for positive polarity at the end of the pulse. All mentioned features are in a good agreement with 2D numerical modeling comparing the $n$ SDBDs of different polarities $[8,9]$. According to numerical modeling, the electric field in the center of a streamer channel of negative polarity is high - about $100 \mathrm{Td}$; for the streamer channel of positive polarity, the field is lower - less than $40 \mathrm{Td}$. The electric field in the streamer front is higher for the positive polarity streamer. 


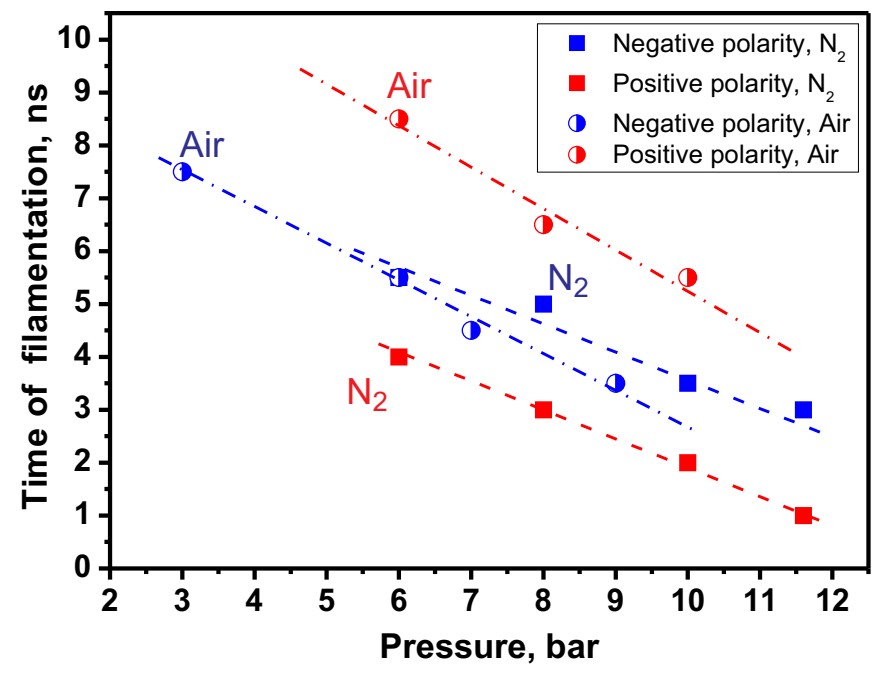

Figure 4. Time instant of filamentation (start of the filaments form the high-voltage electrode) in air and $\mathrm{N}_{2}$ as a function of gas pressure. Applied voltage amplitude for both polarities is $50 \mathrm{kV}$ on the electrode.

At voltages higher than the transition voltage (region $I I$ in figure 3), the filaments exhibit some common features demonstrating a similar nature of filamentary plasmas for both polarities. To demonstrate the similarity, a few experimental techniques have been used.

\subsection{Similar behavior of discharge at different polarity: optical emission, electron density, charge, hydrodynamic perturbations}

The integral emission intensity in the wavelength range $250-800 \mathrm{~nm}$ in the filamentary mode, region $I I$, is 50 times higher than in the streamer mode, region $I$. The optical emission spectrum changes dramatically at the transition point. For any set of parameters belonging to region $I I$, the change of the spectrum in time always correlates with the appearance of filaments. It was necessary to fix the filament position artificially to take the time- and space-resolved optical emission spectra. To do this, the highvoltage electrode with a smooth edge was replaced by a gear of the same external diameter of $20 \mathrm{~mm}$. The gear was selected so that the distance between the teeth was close to the average distance between filaments under the given experimental conditions.

It should be noted that electrodes made from different materials were tested to check how a single-shot nSDBD depends on the material and on the thickness of the electrode. Brass, copper, aluminum and stainless steel electrodes; foil electrodes, $50-200 \mu \mathrm{m}$ in thickness and disk electrodes, $1-2 \mathrm{~mm}$ in thickness with a chamfer angle between 30 and 60 degrees give the same or very similar results. For example, the transition voltage $U_{t r}$ for annealed aluminum or copper electrodes checked for positive polarity discharge in air for the pressure range $5-10$ bar is only $1-2 \mathrm{kV}$ higher than for the non-annealed electrodes [20].

Thus, when a gear was placed instead of the disc electrode, no any visible changes 
of the discharge behavior were observed. The electrode system was trained over a few hundred shots; then a filament was selected, and the discharge chamber rotated so that the filament was aligned with the spectrometer slit. The entrance slit of the spectrometer was equal to $100 \mu \mathrm{m}$ to assure the only filament within the slit area. The camera gate was equal to 5 ns. The spectra were acquired with 100 accumulations. The camera sensitivity was selected to be equal during all the experiments and below the MCP saturation level.

2.2.1. Optical emission The general behavior of positive and negative polarity filaments is similar. From the very beginning, when the discharge propagates as a set of streamers, only the second positive system (SPS) of molecular nitrogen can be detected. When the filaments appear, the $c w$ spectrum is observed. No traces of material of the high-voltage electrode is observed in the spectra. The SPS and the $c w$ components of the same $2 \mathrm{D}$ spectrum are separated in space. The $c w$ spectrum comes from the body of filament, starting from the HV electrode edge (red dashed line) to several mm away, whereas the SPS emission appears at the front of the discharge. That corresponds to the streamer corona that supplies filaments with electrons.

It should be noted that continuum emission in visible and UV-parts of spectra has been observed earlier in high power nanosecond discharges [21-23]. The discharges initiated by $\mathrm{HV}$ pulses in atmospheric pressure air between two metal electrodes separated by a $1-2 \mathrm{~cm}$ air gap. The applied high voltage was about $150-250 \mathrm{kV}$ in amplitude and tens of nanoseconds in pulse duration. Typically, at these conditions the atomic lines corresponding to materials of the electrodes were clearly observed [21].

The presence of atomic lines corresponding to material of the electrode was checked [24] by a direct comparison of the nanosecond discharge and nano- and femtosecond optical breakdowns caused by action of laser generation on the gas. A broadband radiation in a visible range of spectra with no traces of material of the electrode but with clearly detected lines of atomic nitrogen ions was recorded in discharge at $P=1 \mathrm{~atm}$ air in $3 \mathrm{~cm}$ gap under the voltage pulse $90 \mathrm{kV}$ in amplitude [22]. Similar spectra, with atomic nitrogen ions lines dominated over the $c w$ emission, were observed in [25] in the constricted SDBD powered by a burst of unipolar pulses $400 \mu \mathrm{s}$ in duration and $20 \mathrm{kV}$ in amplitude. It should be noted that the most of presented spectrum are socalled review spectrum not calibrated for the spectral sensitivity of the system, and the decay in the UV-region can be caused by the transmission of optics. High-voltage nanosecond discharges excited in open atmosphere at high overvoltages generate [21] high energy electrons and secondary X-ray bremsstrahlung. X-ray doses of the order of magnitude $60-200 \mu \mathrm{R} /$ shot in the vicinity of the electrodes have been reported in [23] for $250-270 \mathrm{kV}$ discharge in atmospheric pressure air.

Quantitative treatment of the observed spectra allows more detailed analysis of a spatial temporal structure of the filament emission. The spectra of the discharge in synthetic air at pressure 6 bar, corrected on the transmission of the spectral system, are presented in figure 5 for negative and in figure 6 for positive polarity of applied pulses. 

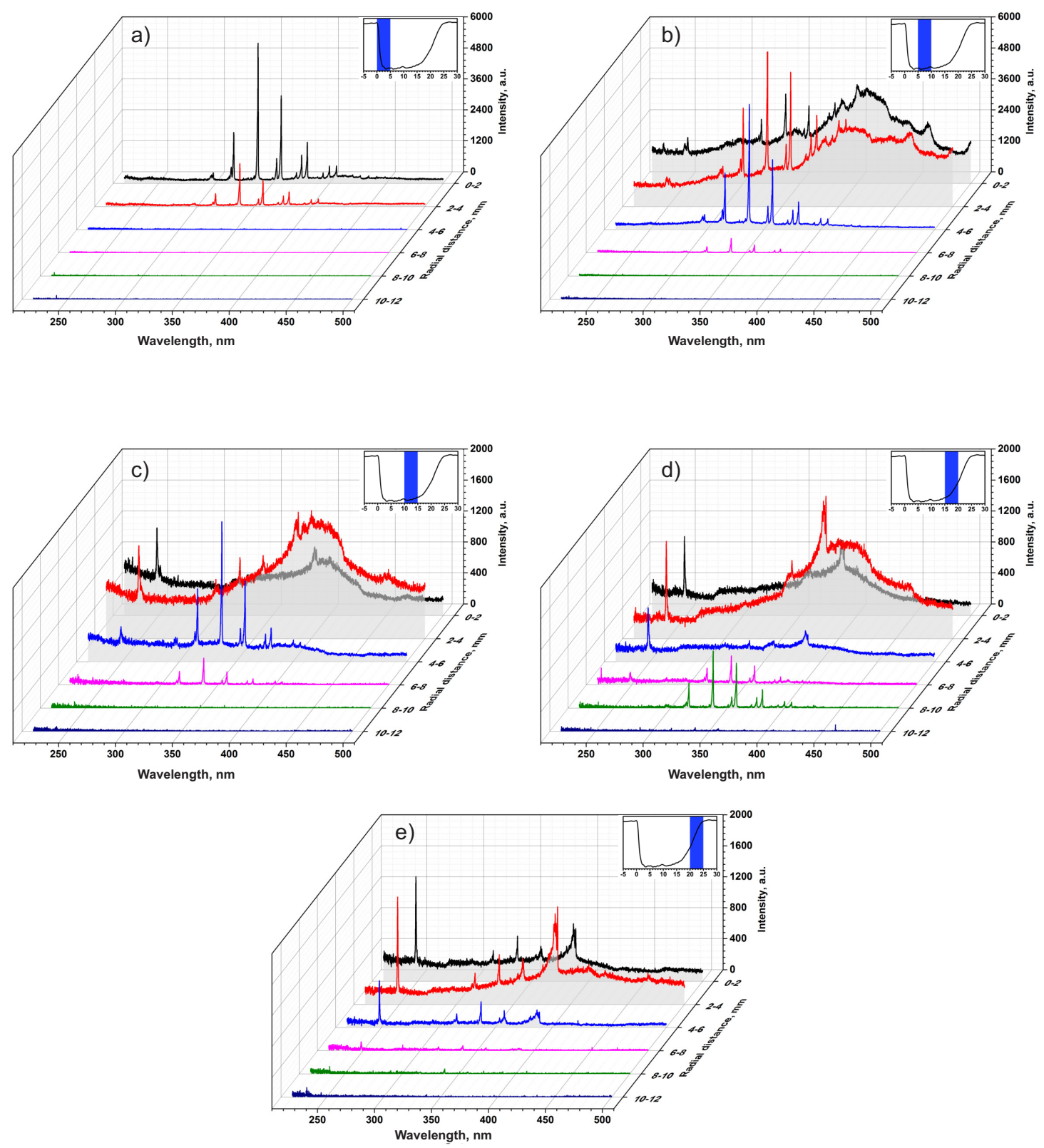

Figure 5. Emission spectra of the negative polarity discharge in air. $P=6$ bar, $U=-50 \mathrm{kV}$. The spectra are corrected to the sensitivity function of the optical system.

Each plot presents the discharge emission as a function of the wavelength and of the distance from the edge of the high-voltage electrode. The spectra are spatially averaged over $2 \mathrm{~mm}$ interval. The corresponding space intervals are indicated on the axis. The insert in the right upper corner of each image provides information about the position of the ICCD gate relative to the waveform of the applied high voltage. The emission of the second positive band of $\mathrm{N}_{2}$, typical for streamer discharge, is clearly seen in the front of the propagating discharge (figures 5 (a) and 6 (a)). The emission of the first 

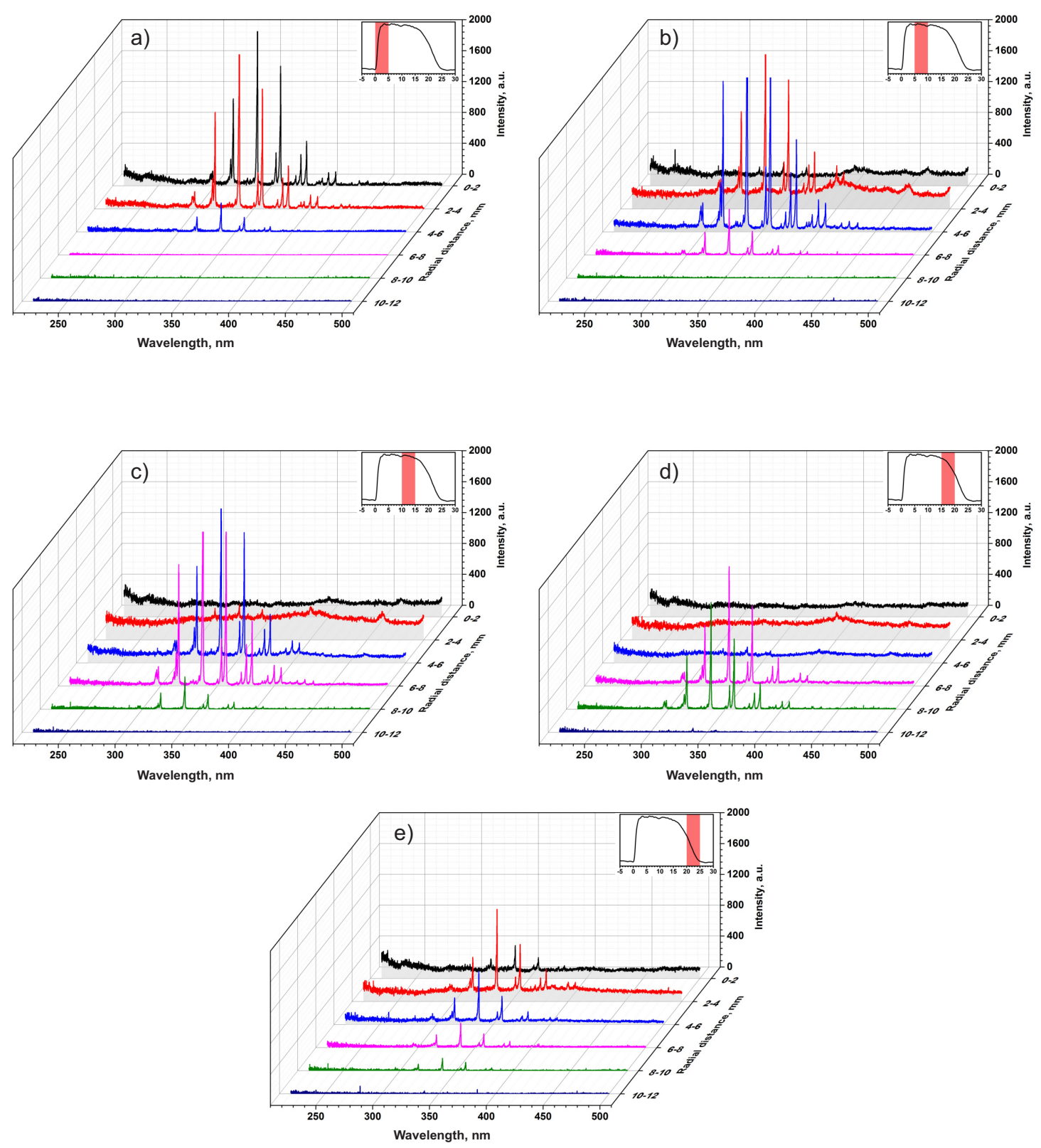

Figure 6. Emission spectra of the positive polarity discharge in air. $P=6$ bar, $U=+50 \mathrm{kV}$. The spectra are corrected to the sensitivity function of the optical system.

negative system of molecular nitrogen at $391.4 \mathrm{~nm}$ is also present in the front of the discharge, indicating high values of electric fields in the streamer corona. Start of the filament is accompanied by appearance of the continuous wavelength spectra near the high-voltage electrode, see the regions $0-2$ and $2-4 \mathrm{~mm}$ in figures 5 (b) and 6 (b)). The streamer corona is clearly seen at the front of the filament $(4-6 \mathrm{~mm}$ from the electrode). The structure when the streamer-like emission is observed at the front of the plasma channel, and the channel itself produces a continuous wavelength emission, 
is typical for the filamentary nSDBD (see figures 5 (c,d) and figures 6 (c.d)). For the negative polarity discharge in air, the intensity of the $c w$ emission is systematically 3-4 times higher than the intensity of the positive polarity discharge, and the shape is somewhat different from the shape of the $c w$ emission of the positive discharge. At the trailing edge of applied pulse (figures 5 (e) and 6 (e)) the second ionization wave starts from the HV electrode and propagates on the trace of the filament. The second ionization wave contains emission of the second positive system of molecular nitrogen indicating on the presence of the electric field comparable to the fields in the discharge front. Emission of the SPS systems both in the discharge front and from the trailing edge of the voltage pulse at any observed experimental conditions, corresponds to a low rotational temperature: between 300 and $500 \mathrm{~K}$. As far as in the filaments no SPS emission is observed, two options are possible and need additional experimental verification [26]: (i) the electric field in the filament is lower than needed for the SPS excitation; (ii) almost complete dissociation takes place in the filament and the emission of the SPS at the trailing edge comes from the streamer zone around the filament.

Quantitative comparable analysis of intensity of the $c w$ radiation and of the SPS bands intensity led to the conclusion [?] that the observed $c w$ radiation is a recombination radiation caused by a high electron density, on the order of $n_{e} \approx$ $10^{18}-10^{19} \mathrm{~cm}^{-3}$ in the discharge.
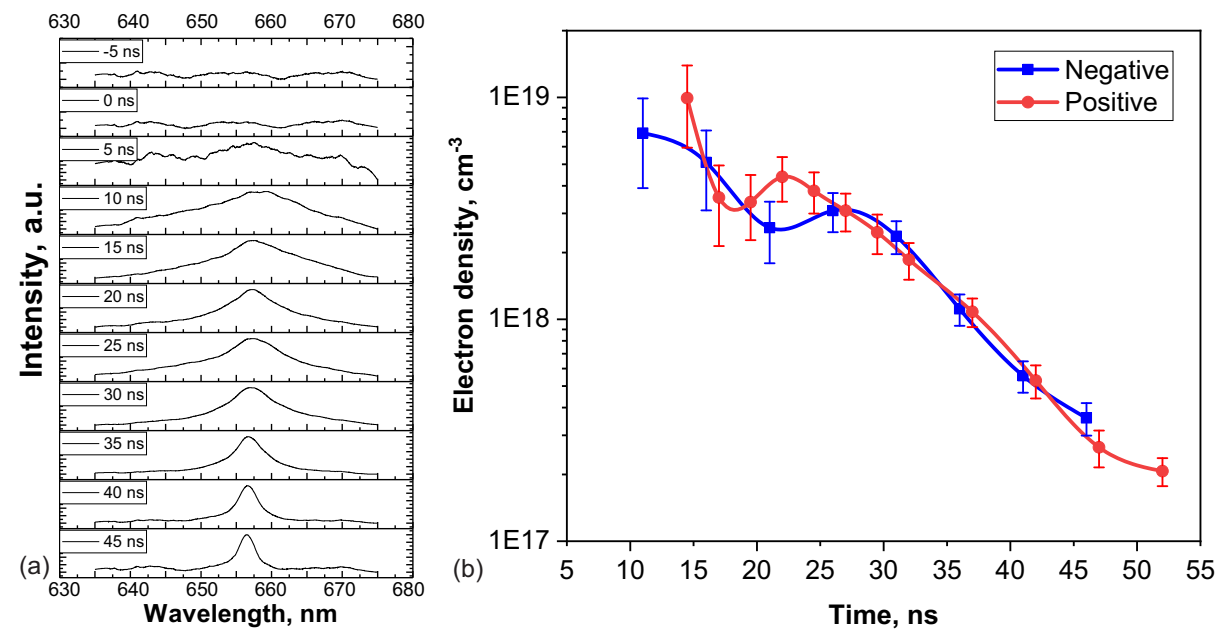

Figure 7. Measurements of electron density in the discharge $\mathrm{H}_{2}: \mathrm{N}_{2}=1: 4$ mixture at $P=8$ bar: (a) spectra of $\mathrm{H}_{\alpha}$ line $(656.5 \mathrm{~nm}$ ) at different time instances; (b) behavior of electron density $v s$ time at negative and positive polarities.

2.2.2. Electron density and surface charge The electron density was measured in discharge in $\mathrm{H}_{2}: \mathrm{N}_{2}=1: 4$ mixture at $P=8$ bar; hydrogen atomic $\mathrm{H}_{\alpha}$ line at $\lambda=656.3 \mathrm{~nm}$ was analyzed for both polarities of the high voltage pulse. Continuous spectra were similar to the spectra observed in air. The time instant of appearance of the emission 
of atoms correlated with appearance of the $c w$ spectra. Figure 7 (a) shows the row data of $\mathrm{H}_{\alpha}$ line appearance in the negative polarity discharge. Camera gate for each spectra was equal to $5 \mathrm{~ns}$. The width of the line obviously decreases with time: the widest line was detected at time instant of $10 \mathrm{~ns}$, the FWHM being equal about $20 \mathrm{~nm}$. The FWHM almost does not change with the distance from the high-voltage electrode and with the voltage amplitude. Electron density as a function of time is presented in figure 7 (b). High values of electron density, about $n_{e} \approx(6-8) \cdot 10^{18} \mathrm{~cm}^{-3}$ are obtained for the discharge phase; the decay of the electron density in the afterglow, by a factor of 2 during $5 \mathrm{~ns}$, is slow. No distinctive difference is observed between behavior of the electron density for negative and positive polarity discharge. It was suggested [26] that such a slow decay of the electron density is due to fact that plasma in the filaments is close to local thermal equilibrium (LTE); the gas temperature in the discharge is a few electronvolts, and the dynamics of plasma decay is determined mainly by gas cooling. This idea is developing in [27], where a kinetic model is suggested to provide a sharp, parts of nanoseconds, transition from streamer to filamentary plasma.

Analysis of surface charge per streamer and per filament shows that for both polarities, the charge per filament accumulated on the surface is at least one order of magnitude higher than the charge per streamer [28]. A set of supplementary experiments where the high-voltage electrode was made of a $\mathrm{Cu}$ foil and contained the teeth at the different distances showed that in the case when the teeth were separated by the distance higher than the distance between the filaments naturally obtained at the smooth electrode edge, a few, typically three, filaments started from each tooth, at the angles providing a maximum separation in space of the staring filaments. This fact confirms that a regular structure of the filaments is due to significant charge of each channel and that it is the charge defining the distance between the filaments in each particular case.

As far as (i) the filaments start from the edge of the high-voltage electrode; (ii) the properties of plasma in the filaments are similar for both polarities of high voltage pulse, it is reasonable to assume that the start of the filaments and the streamer-to-filament transition depend upon the high-voltage polarity and described by different physics, but once started, filaments of both polarities produce quite similar plasma and can be described by the same physical model.

Paper [27] suggests that one of important conditions for development of filament is an increased electron density in the vicinity ot the high-voltage electrode. In the case of the cathode a reason of formation of the high electron density near the electrode can be a field emission instability. Questions about the instability of the anode layer,possible formation of anode spots and the role of photoionization in sustaining the streamers in high pressure SDBD remain open.

When the high electron density is produced near the high-voltage electrode, the potential of the electrode is "transferred" in the bulk of plasma; the filament propagates in the bulk of the streamer plasma as a fast ionization wave (FIW) or the wave of a gradient of the electrical potential

Kinetic model, describing appearance of the high observed electron density (see [27] 
for more details), is common for both polarities of the discharge and assumes that high electric fields (om the level of $200 \mathrm{Td}$ and higher) exists during at least parts of nanoseconds. In this case, at high values of specific delivered energy at high gas density, reactions of step-wise ionization with participation of electronically excited levels will provide high, $10^{18}-10^{19} \mathrm{~cm}^{-3}$, electron densities. Significant heat release in reactions of recombination and reactions of dissociation in collisions with electronically excited species will result in high, tens of kK per parts of nanoseconds, heating and transition to filamentary polasma to the state close to the local thermal equilibrium. The density of atomic species in the observed filamentary plasma should be also extremely high. The LTE explains a slow decay of the electron density following the cooling down of the filament.

2.2.3. Hydrodynamic perturbations Schlieren technique has been used for diagnostics of hydrodynamic and thermal effects of nSDBD. Typical schlieren images of hydrodynamic perturbations caused by streamer and filamentary discharges in air are presented in figure 8. The total energy deposited into discharge is equal to $9 \mathrm{~mJ}$ in the case of the streamer discharge at $P=4$ bar and $U=-25 \mathrm{kV}$ (figure 8 (a)) and to $49 \mathrm{~mJ}$ in the case of the filamentary discharge at $P=6$ bar and $U=-50 \mathrm{kV}$ (figure 8 (b)). For both streamer and filamentary discharges, the hydrodynamic perturbations provided by separate channels, streamers or filaments, merge together. In the case of the streamer discharge the resulting front of a hydrodynamic perturbation is a disc with an empty space at the place of the high-voltage electrode propagating in the direction perpendicular to the surface of the dielectric (from right to left in the figure), the external radius correspond to the maximum length of the streamer propagation. For filamentary discharge the picture is similar but the contrast is stronger and the cylindrical waves produced by separate filaments are clearly seen even at tens of microseconds after the discharge. For streamer discharge, the perturbation decays reaching the upper end plate of the discharge chamber at about $80 \mu \mathrm{s}$; for filamentary discharge, a series of reflection without significant lost of contrast is observed up to $500 \mu \mathrm{s}$. Described behaviour is similar to positive and negative polarity filamentary discharge.

The velocities of the compression waves in the direction perpendicular to the dielectric are presented in figure 9 a. During a few microseconds after the discharge (see the inert to the figure) the velocity of the formed perturbation slightly exceeds the sound speed, $M=1.1-1.3$, for streamer discharge, and comprises $M \approx 1.5-1.7$ for filamentary discharge; later, the compression wave propagates practically with a speed of sound. The decay of hydrodynamic perturbation intensity for three selected cases and presented in figure $9 \mathrm{~b}$. The relative contrast $C(t)=\left(I_{\text {front }}(t)-I_{0}\right) / I_{0}$, where $I_{0}$ is an intensity of the background light at fixed cutoff knife position, is shown for streamers of both polarities $( \pm 25 \mathrm{kV})$ and for filaments of negative polarity $(-50 \mathrm{kV})$. It is clearly seen that the decay of the wave during first $60 \mu \mathrm{s}$ is about $80 \%$ for streamers and only $20 \%$ for filaments.

Because of a complex 3D morphology of the filamentary discharge in cylindrical 
Filamentary nanosecond SDBD. Comparison of both polarities.

(a)
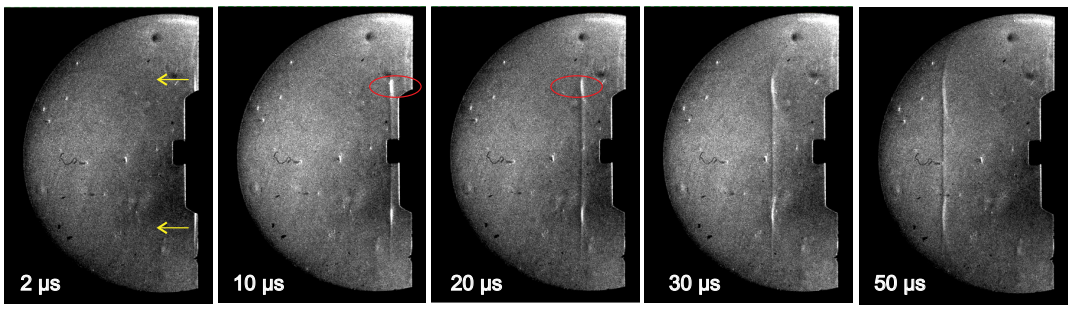

(b)
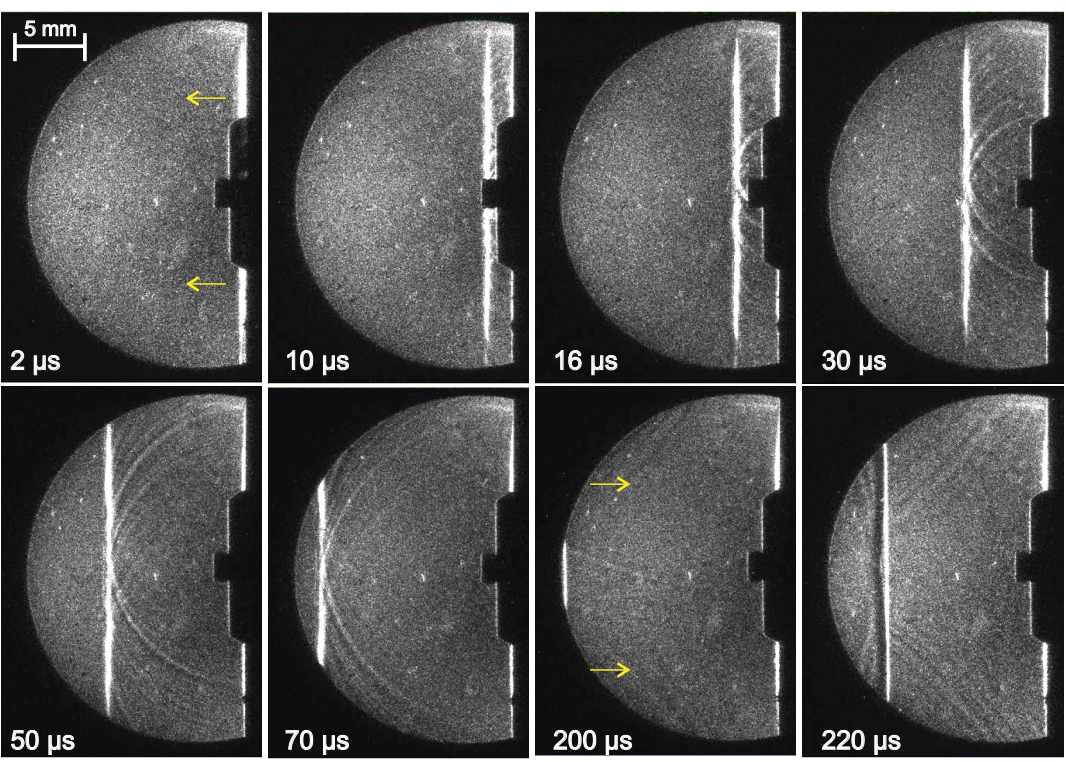

Figure 8. Schlieren images of the compression wave in air; ICCD camera gate is 200 ns: (a) $P=4$ bar, $U=-25 \mathrm{kV}$; (b) $P=6$ bar, $U=-50 \mathrm{kV}$.

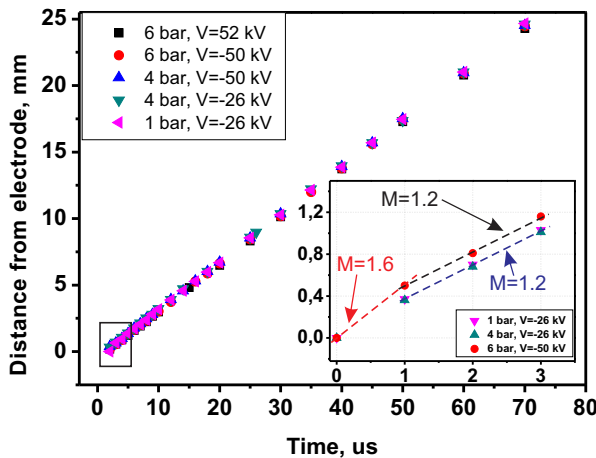

a)

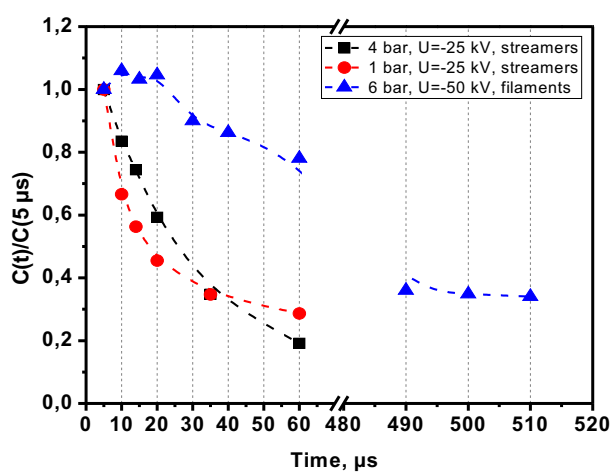

b)

Figure 9. (a) Velocities of shock wave propagation at different pressures and applied voltages; (b) Contrast dynamics in the front of compression waves.

configuration, calculation of the exact value of pressure is complicated. Nevertheless, it is possible to make the estimates assuming that the compression wave has a cylindrical symmetry and to analyze some hydrodynamic effects from the "summary" perturbation. 
Figure 10 provides a comparison between schlieren images at two polarities of the applied voltage for the filamentary discharge in air at $P=6$ bar and $U= \pm 50 \mathrm{kV}$. The $\mathrm{N}$-shape of the profiles is clearly seen. The total delivered energies are close being equal to $49 \mathrm{~mJ}$ for the negative polarity discharge and $38 \mathrm{~mJ}$ for the positive polarity discharge. The intensities of the waves are also similar. High contrast of the schlieren images for the filamentary discharge proves that the specific energy release in the filaments is high, and the uniform distribution of contrast along the radius of the discharge system confirms that the distribution of plasma parameters along the filament should be uniform. This fact is in a good agreement with behavior of the optical emission and of the electron density along the filaments.

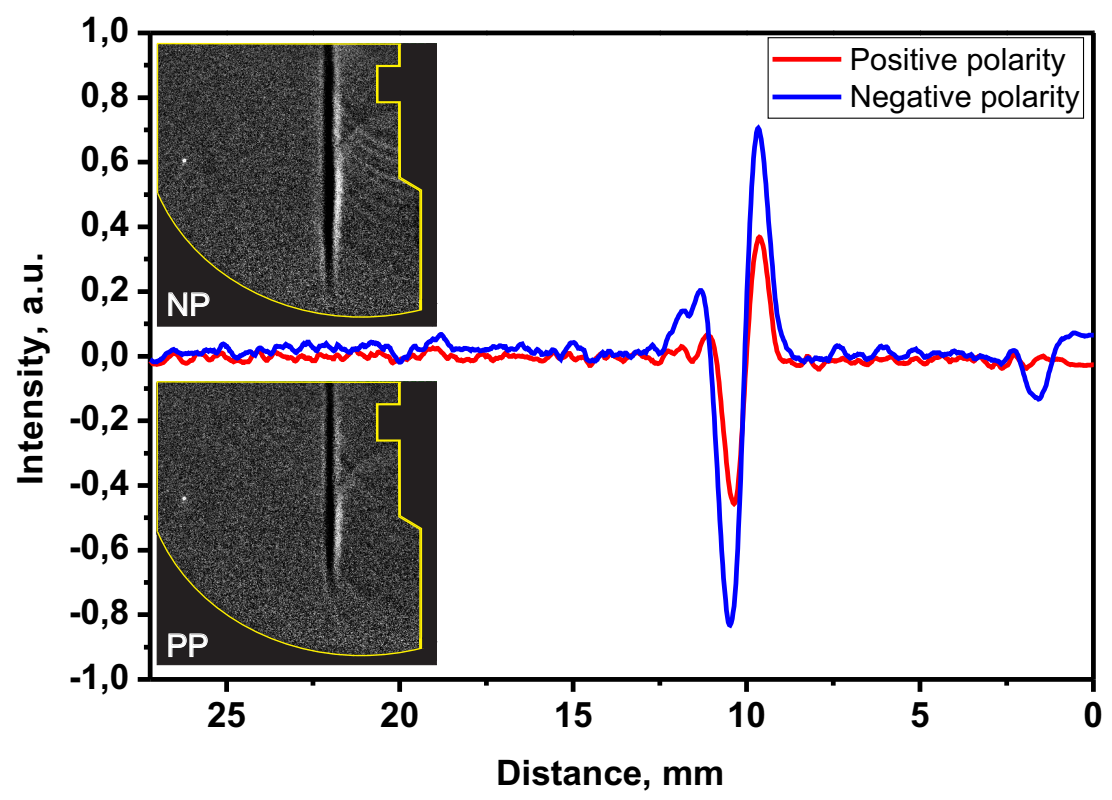

Figure 10. Schlieren images of the compression wave and intensity profiles of the images in the direction perpendicular to the plane of the dielectric in air; $P=6$ bar, $U= \pm 50 \mathrm{kV}$. NP is for negative polarity and PP is for positive polarity. ICCD camera gate is $200 \mathrm{~ns}$.

The values of pressure in the front of the compression wave were compared for different experimental conditions. Four cases were considered: 1) filamentary discharge at $P=6$ bar and $U=-50 \mathrm{kV} ; 2$ ) filamentary discharge at $P=6$ bar and $U=+50 \mathrm{kV}$; 3) streamer discharge at $P=6$ bar and $U=-25 \mathrm{kV}$; $)$ streamer discharge at $P=1$ bar and $U=-25 \mathrm{kV}$. The pressure profiles in arbitrary units were restored using the following equation:

$$
P(r) \sim \int_{r}^{+\infty} \frac{d}{d x}\left(\int_{x}^{+\infty} I\left(l^{\prime}\right) d l^{\prime}\right) \frac{d x}{\sqrt{x^{2}-r^{2}}} .
$$

The profiles were averaged over the wavefront thickness as it is shown in figure $11 \mathrm{a}$. The segment near the maximum amplitude was selected. The dark region in the insert 


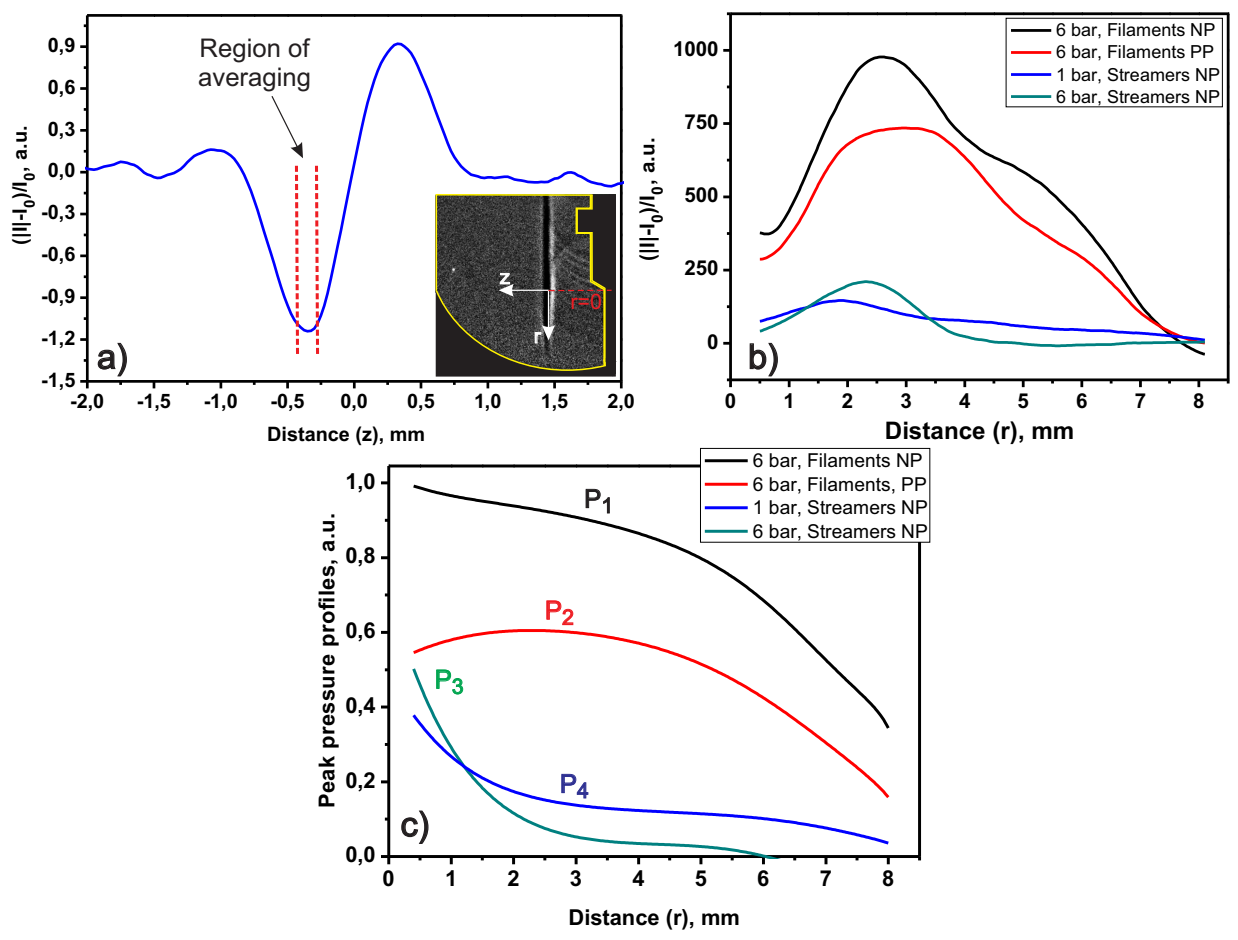

Figure 11. (a) N-wave structure. Representation of the region of averaging for pressure analysis; (b) Radial profiles of the contrast. $|I|$ is an absolute value of intensity of the light in arbitrary units; (c) pressure profiles in arbitrary units (in radial direction)

of schlieren image (figure 11 a) corresponds to the pressure surplus in the wavefront. The radial normalized on $I_{0}$ intensity profiles are plotted in figure $11 \mathrm{~b}$. The profiles are plotted for time instant $25 \mu \mathrm{s}$ after the discharge. Figure $11 \mathrm{c}$ demonstrates the pressure profiles in radial direction. It is clearly seen that both the surplace pressure behavior and absolute values of pressures are different depending on gas pressure and discharge structure. The ratio of maximum pressures for considered cases is $P_{1}^{\max } / P_{2}^{\max } / P_{3}^{\max } / P_{4}^{\max }=2.7 / 1.6 / 1.3 / 1$.

In addition to the traveling wave, another hydrodynamic perturbation appears in the system: tens and hundreds microseconds after the filamentary nSDBD, a heated region is produced near the dielectric. The region was visualized in 6 bar air by schlieren technique with a long gate of the ICCD, $10 \mu$ s (figure 12 (a)). The blurred image of the $\mathrm{N}$-wave moving from the dielectric is seen at the first frame $(5 \mu \mathrm{s})$. The turbulization of the heated region starts from 100-200 $\mu$ s; the thickness of the region increases in time (figure $12(\mathrm{~b})$ ) from 1 to $5 \mathrm{~mm}$ between $5 \mu$ s and $1 \mathrm{~ms}$.

As far as the trace of each filament is a heated region containing atomic species and radicals, and tens of filaments about $1 \mathrm{~cm}$ long can be produced in the discharge, the filamentary nanosecond surface dielectric barrier discharge is an efficient tool for plasma-assisted distributed ignition of combustible mixtures. The traces of the filaments initiate multiple combustion waves and their interaction leads to efficient ignition of the 


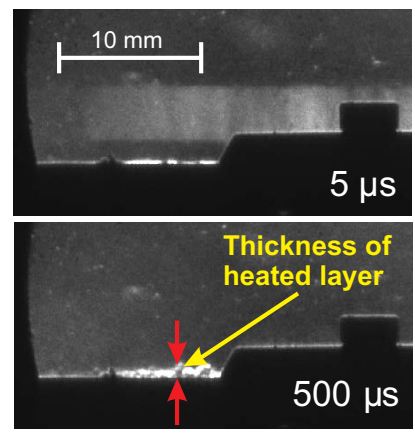

a)

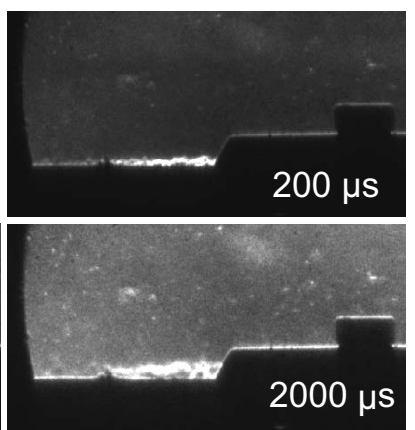

b)

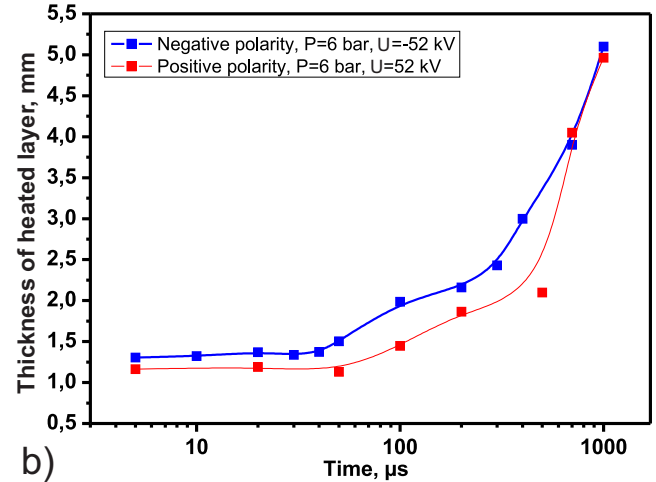

Figure 12. (a) schlieren images of the near electrode heated region; (b) thickness of the near-electrode perturbation as a function of time. Air, $P=6$ bar, $|U|=50 \mathrm{kV}$, camera gate $10 \mu \mathrm{s}$.

mixture throughout the volume. Additionally the heated region in close proximity of the dielectric layer initiates a turbulization of the combustion wave front and accelerates its propagation [13].

\section{Conclusions}

The streamer-to-filament transition and properties of the filamentary mode of a nanosecond surface dielectric barrier discharge were studied over a wide range of pressures $(1-12$ bar) and voltage amplitudes on the high voltage electrode $(20-60 \mathrm{kV})$ for different gases (air, $\mathrm{N}_{2}, \mathrm{~N}_{2}: \mathrm{O}_{2}$ and $\mathrm{N}_{2}: \mathrm{H}_{2}$ mixtures). Discharges of negative and positive polarities were compared. Overall, we can conclude that the streamer-tofilament transition is a general feature for any mixtures containing molecular gases.

The transition is observed at high pressures and voltages in a single-shot experiment at the rising front or on the plateau of the high-voltage pulse. At the moment of transition, regardless of the polarity, several filaments start from the high voltage electrode. In the following $1-3$ ns a regular pattern of filaments is formed around the electrode. Streamers propagate in the radial direction with a velocity of a few millimeters per nanosecond on the front of the pulse; the velocity of filament propagation is at least a few times lower. Filaments can reach lengths of about $1 \mathrm{~cm}$ at pressures around 10 bar, filling the dielectric surface with a regular pattern of plasma channels. The distance between the filaments is a few times higher than the distance between streamers in a nanosecond SDBD.

The streamer-to-filament transition is a function of the applied high voltage and the gas pressure. The transition voltage is not sensitive to the shape and material of the high-voltage electrode but sensitive to the applied voltage polarity. When the negative polarity is applied, the transition voltage slightly changes with pressure (e.g. between 35 and $30 \mathrm{kV}$ for nitrogen in the pressure range between 4 and 10 bar) and with gas mixture composition (e.g. between 37 and $32.5 \mathrm{kV}$ for $5 \%$ of molecular oxygen in nitrogen in the pressure range between 4 and 10 bar). At the positive polarity, the 
transition voltage changes significantly with pressure, from 41 to $20-25 \mathrm{kV}$ for nitrogen in the same pressure range. The effect of molecular oxygen addition is extremely strong: $1 \%$ of oxygen at 4 bar increases the transition voltage from $41 \mathrm{kV}$ to $52 \mathrm{kV}$.

Despite the different conditions for the streamer-to-filament transition, the filaments' plasma exhibits similar features for both polarities of applied pulses. The electrical current reaches values up to several amperes per filament, an order of magnitude higher than the current in streamers. Before the streamer-to-filament transition, mainly nitrogen molecular bands are observed in the optical emission spectra, which is typical for low temperature nanosecond plasmas. Afterwards, the emission changes abruptly to the continuous wavelength spectra in UV and visible range, the molecular bands of nitrogen disappear, and the observed atomic lines $\left(\mathrm{H}, \mathrm{N}^{+}, \mathrm{O}\right)$ are broadened. The electron density derived from the $\mathrm{H}_{\alpha}$ emission is high in the discharge $\left(n_{e}=10^{18}-10^{19} \mathrm{~cm}^{-3}\right.$ ) and decays slowly (within tens of nanoseconds) in the afterglow. The electrical charge on the dielectric is an order of magnitude higher for filaments than for streamers. The filamentary discharge produces a long-lasting (several ms) hydrodynamic perturbation, regardless of the applied polarity, which indicates a high specific energy release per filament. The similarity between plasma filaments created by either polarity of applied pulse allows for coherence in the approaches used to study the physics behind a filamentary discharge.

\section{Acknowledgements}

The work was partially supported by LabEx Plas@Par, French National Research Agency (ASPEN Project) and the French-Russian international laboratory LIA KaPPA "Kinetics and physics of pulsed plasmas and their afterglow" (CNRS financial and organization support). The support of China Scholarship Council (CSC) for Chenyang

Ding is gratefully acknowledged. The authors are thankful to Ali Mahjoub and Bruno Dufour for engineering support.

\section{References}

[1] Opaits D, Roupassov D, Starikovskaia S, Starikovskii A, Zavialov I and Saddoughi S 2005 Plasma control of boundary layer using low-temperature non-equilibrium plasma of gas discharge 43rd AIAA Aerospace Sciences Meeting and Exhibit p 1180

[2] Starikovskii A Y, Nikipelov A, Nudnova M and Roupassov D 2009 SDBD plasma actuator with nanosecond pulse-periodic discharge Plasma Sources Science and Technology 18034015

[3] Leonov S, Opaits D, Miles R and Soloviev V 2010 Time-resolved measurements of plasma-induced momentum in air and nitrogen under dielectric barrier discharge actuation Physics of Plasmas 17113505

[4] Rethmel C, Little J, Takashima K, Sinha A, Adamovich I and Samimy M 2011 Flow separation control using nanosecond pulse driven DBD plasma actuators International Journal of Flow Control 3 213-232 ISSN 1756-8250

[5] Bayoda K, Benard N and Moreau E 2015 Nanosecond pulsed sliding dielectric barrier discharge plasma actuator for airflow control: electrical, optical, and mechanical characteristics Journal of Applied Physics 118063301 
Filamentary nanosecond SDBD. Comparison of both polarities.

[6] Pancheshnyi S, Starikovskaia S and Starikovskii A Y 2000 Collisional deactivation of $\mathrm{N}_{2}\left(\mathrm{C}^{3} \Pi_{u}\right.$, $\mathrm{v}=0,1,2,3)$ states by $\mathrm{N}_{2}, \mathrm{O}_{2}, \mathrm{H}_{2}$ and $\mathrm{H}_{2} \mathrm{O}$ molecules Chemical Physics 262 349-357

[7] Soloviev V and Krivtsov V 2009 Surface barrier discharge modelling for aerodynamic applications Journal of Physics D: Applied Physics 42125208

[8] Babaeva N Y, Tereshonok D V and Naidis G V 2016 Fluid and hybrid modeling of nanosecond surface discharges: effect of polarity and secondary electrons emission Plasma Sources Science and Technology 25044008

[9] Zhu Y, Shcherbanev S, Baron B and Starikovskaia S 2017 Nanosecond surface dielectric barrier discharge in atmospheric pressure air: I. measurements and 2D modeling of morphology, propagation and hydrodynamic perturbations Plasma Sources Science and Technology $\mathbf{2 6} 125004$

[10] Starikovskaia S, Allegraud K, Guaitella O, Kosarev I, Mintusov E, Pendleton S J, Popov N, Sagulenko P and Rousseau A 2010 Surface discharges: possible applications for plasma-assisted ignition and electric field measurements 48th AIAA Aerospace Sciences Meeting Including the New Horizons Forum and Aerospace Exposition p 1587

[11] Kosarev I, Khorunzhenko V, Mintoussov E, Sagulenko P, Popov N and Starikovskaia S 2012 A nanosecond surface dielectric barrier discharge at elevated pressures: time-resolved electric field and efficiency of initiation of combustion Plasma Sources Science and Technology 21045012

[12] Stepanyan S, Starikovskiy A, Popov N and Starikovskaia S 2014 A nanosecond surface dielectric barrier discharge in air at high pressures and different polarities of applied pulses: transition to filamentary mode Plasma Sources Science and Technology 23045003

[13] Shcherbanev S A, Popov N A and Starikovskaia S M 2017 Ignition of high pressure lean $\mathrm{H}_{2}$ : air mixtures along the multiple channels of nanosecond surface discharge Combustion and Flame $176272-284$

[14] Shcherbanev S, Khomenko A Y, Stepanyan S, Popov N and Starikovskaia S 2017 Optical emission spectrum of filamentary nanosecond surface dielectric barrier discharge Plasma Sources Science and Technology 26 02LT01

[15] Van der Horst R, Verreycken T, Van Veldhuizen E and Bruggeman P 2012 Time-resolved optical emission spectroscopy of nanosecond pulsed discharges in atmospheric-pressure $\mathrm{N}_{2}$ and $\mathrm{N}_{2} / \mathrm{H}_{2} \mathrm{O}$ mixtures Journal of Physics D: Applied Physics 45345201

[16] Lo A, Cessou A, Lacour C, Lecordier B, Boubert P, Xu D, Laux C and Vervisch P 2017 Streamerto-spark transition initiated by a nanosecond overvoltage pulsed discharge in air Plasma Sources Science and Technology $\mathbf{2 6} 045012$

[17] Orrière T, Moreau E and Pai D Z 2018 Ionization and recombination in nanosecond repetitively pulsed microplasmas in air at atmospheric pressure Journal of Physics D: Applied Physics $\mathbf{5 1}$ 494002

[18] Anikin N, Starikovskaia S and Starikovskii A Y 2002 Polarity effect of applied pulse voltage on the development of uniform nanosecond gas breakdown Journal of Physics D: Applied Physics 352785

[19] Xu D, Shneider M, Lacoste D and Laux C 2014 Thermal and hydrodynamic effects of nanosecond discharges in atmospheric pressure air Journal of Physics D: Applied Physics 47235202

[20] Selivonin I, Moralev I, Shcherbanev S, Alicherif M and Starikovskaya S 2018 Dependence of ignition and constriction voltage of surface barrier discharge from pressure and material of corona electrode XLV Zvenigorod International Conference on Plasma Physics and Controlled Fusion (Zvenigorod, Russia)

[21] Babich L P, Loiko T and Tsukerman V 1990 High-voltage nanosecond discharge in a dense gas at a high overvoltage with runaway electrons Physics-Uspekhi 33 521-540

[22] Shao T, Tarasenko V F, Zhang C, Lomaev M I, Sorokin D A, Yan P, Kozyrev A V and Baksht E K 2012 Spark discharge formation in an inhomogeneous electric field under conditions of runaway electron generation Journal of Applied Physics 111023304

[23] Babich L and Loiko T 2010 Peculiarities of detecting pulses of runaway electrons and x-rays generated by high-voltage nanosecond discharges in open atmosphere Plasma physics reports $\mathbf{3 6}$ 
263-270

[24] Prokopyev V, Ivanov N, Krivonosenko D and Losev V 2014 Investigation of the elementary physical processes in plasma of filamentation and optical breakdown regions accompanying the propagation of the femtosecond laser pulse with wavelength of $950 \mathrm{~nm}$ in air at atmospheric pressure Russian Physics Journal 56 1274-1280

[25] Houpt A W and Leonov S B 2016 Charge transfer in constricted form of surface barrier discharge at atmospheric pressure Journal of Thermophysics and Heat Transfer 31 145-153

[26] Shcherbanev S, Popov N and Starikovskaia S 2018 Multi-point nanosecond surface discharge at high pressures for plasma assisted ignition: spectroscopy study of the filamentary phase 2018 AIAA Aerospace Sciences Meeting p 0686

[27] Shcherbanev S, Ding C, Starikovskaia S and Popov N 2019 Filamentary nanosecond surface dielectric barrier discharge. Plasma properties in the filaments Plasma Sources Science and Technology Submitted PSST-102956

[28] Shcherbanev S, Williams J, Popov N and Starikovskaia S 2017 On filamentary nanosecond surface dielectric barrier discharge for flame initiation Proc. of the 23rd International Symposium on Plasma Chemistry (ISPC) (Montreal, Canada) july 30 - August 4 